\title{
Temporary negative-ion effects on photon emission from free zinc atoms excited by electron impact
}

\author{
S. A. Napier \\ Centre for Atomic Molecular and Surface Physics \\ D. Cvejanović, J. F. Williams, ${ }^{*}$ and L. Pravica \\ Centre for Atomic Molecular and Surface Physics and ARC Centre for Antimatter-Matter Studies, Physics Department, \\ University of Western Australia, Perth 6009, Australia \\ (Received 27 March 2008; published 4 August 2008)
}

\begin{abstract}
Temporary negative-ion formation has been investigated by observing photon emission from electronimpact-excited $4 s 4 p{ }^{3} P_{1}, 4 s 4 p{ }^{1} P_{1}, 4 s 4 d{ }^{3} D_{1,2,3}, 4 s 4 d{ }^{1} D_{2}$, and $4 s 5 d{ }^{3} D_{1,2,3}$ states of zinc. Photon excitation functions are presented for incident electron energies from the first excited state threshold $(4.003 \mathrm{eV})$ to the first ionization potential $(9.394 \mathrm{eV})$. The energies of isolated negative-ion resonance structures have been determined by fitting Fano profiles. These energies, and those of key features in the complicated overlapping resonance profiles, are compared with theory and previous experiments. The strength of resonance contributions, and which structures are dominant, depends on the spin and orbital angular momentum properties of the observed state.
\end{abstract}

DOI: 10.1103/PhysRevA.78.022702

PACS number(s): 34.80.Dp

\section{INTRODUCTION}

Recent studies of the interactions between many-electron atoms and incident electrons or photons have investigated electron correlations, channel coupling, relativistic effects, and the optimal methods for their observation. Knowledge of these effects is essential because they are important in determining how the structure and scattering dynamics of complex atoms vary across the periodic table. Applications of that knowledge will result in more accurate models of plasmas and discharges, for example.

Models based on the assumption of inert core electrons successfully describe some, but not all, experimental observations for quasi-one and -two electron atoms. An interdependence of $3 d$ core and outermost $4 s$ shells due to electron correlations and variable nuclear charge screening effects is apparent in the $3 d^{10} 4 s$ ground state electron configuration of copper $(Z=29)$. A $3 d^{9} 4 s^{2}$ ground state configuration is expected from the trend across the periodic table, but this is in fact an excited state of copper, $1.389 \mathrm{eV}$ above the ground state. Zinc $(Z=30)$ also has an occupied $4 s$ shell outside a full $3 d$ shell in its ground state configuration of $3 d^{10} 4 s^{2}$. Since the wave function of an unfilled $d$ orbital penetrates further into a core containing a filled $d$ orbital, for zinc it is reasonable to expect significant correlations between inner $3 d$, outer $4 s$, and excited $n d$ electrons. This may result in the formation of temporary negative ions with a $4 s n d n^{\prime} l^{\prime}$ electron configuration, and indeed, the $n=4,5 n^{\prime}=5,6,7$ resonances are predicted by theory [1]. The present experiment investigates these electron correlations by measuring the effect temporary negative ion formation has on the electronimpact excitation of $4 s 4 p^{1,3} \mathrm{P}$ and, in particular, $4 s n d^{1,3} \mathrm{D}$ states of zinc.

A comprehensive review of negative-ion resonances, including the IIB metal atoms, is presented by Buckman and

\footnotetext{
*jfw@cyllene.uwa.edu.au
}

Clark [2]. A more recent investigation and discussion relevant to $\mathrm{Zn}, \mathrm{Cd}$, and $\mathrm{Hg}$ was published by Sullivan et al. [3]. Some similarities exist in the neutral atomic and negative-ion spectra for these atoms, but significant differences are observed primarily due to the increasing magnitude of spinorbit interactions with increasing $Z$. In mercury, a large spinorbit interaction separates the lowest $5 d^{10} 6 s 6 p^{3} P_{0,1,2}$ neutral excited states by $793 \mathrm{meV}$, and pushes some of the core excited $5 d^{9} 6 s^{2} 6 p$ states below the first ionization threshold of $10.44 \mathrm{eV}$. For example, the $5 d^{9} 6 s^{2}\left[{ }^{2} D_{5 / 2}\right]^{3} P_{2}$ state is located at $8.54 \mathrm{eV}$. Such fine structure effects in $\mathrm{Hg}$ negativeion resonances are large relative to zinc, and have been resolved by a number of electron-impact studies (for example, Newman et al. [4]).

When compared to $\mathrm{He}$ and $\mathrm{Hg}$, there have been relatively few zinc studies and there is little information indicating when correlation and relativistic effects need to be taken into account for this medium-sized atom. Fine structure splitting in zinc is much smaller than in mercury, with the lowest neutral excited states $4 s 4 p{ }^{3} P_{0,1,2}$ separated by $72 \mathrm{meV}$ as compared to the $793 \mathrm{meV}$ separation between the $6 s 6 p{ }^{3} P_{0,1,2}$ states of mercury. Unlike mercury, the $3 d^{9} 4 s^{2} n l$ states of zinc formed by exciting a core electron from the outermost $d$ shell all lie above the first ionization threshold of $9.394 \mathrm{eV}$. For zinc, interactions with the core will not be dominated by spin-orbit effects as they are in mercury, and there will be a more balanced interplay between magnetic, electrostatic, and exchange interactions. A comprehensive study of negative-ion resonances is expected to be sensitive both to spin-dependent interactions and core screening effects.

Spin effects in zinc associated with $3 d$-core electron excitation have been observed with integrated Stokes parameter measurements of decay photons from the $4 s 4 d{ }^{1} D_{2}$ state excited by spin-polarized incident electrons [5]. That study revealed and disentangled spin-orbit and exchange effects due to negative-ion resonances above the ionization threshold. 
However, there has been no modeling of negative-ion resonances involving the excitation of one $3 d$ electron and the existing theoretical negative-ion resonance data are limited to below the ionization threshold.

Several experimental studies have been conducted by the Uzghorod group. Their measurements include optical excitation functions for the $4 s 4 p{ }^{3} P_{1}, 4 s 5 s{ }^{3} S_{1}, 4 s 5 d{ }^{3} D_{1,2,3}$ [6] and 4 snd ${ }^{1} D_{2}, n=4,5,6[7]$ states, as well as cross sections for total scattering, ionization, and $4 s 4 p{ }^{3,1} P$-state excitation [8]. Their work maps the negative-ion resonances in zinc, but their excitation functions are limited by the energy resolution. Transmission measurements by Burrow et al. up to $\sim 6.8 \mathrm{eV}$ [9] have been extended to $10 \mathrm{eV}$ by Sullivan et al. [3]. They identify the energies of observed structures but there is no information on the decay channels.

Two theoretical models have been used by Zatsarinny and Bartschat [1] to calculate integral electron-impact excitation cross sections for zinc. The first was a standard $R$-matrix (RM) approach with orthogonal orbitals [3] and the second a $B$-spline $R$-matrix (BSRM) method which used termdependent, nonorthogonal target orbitals [10]. While both models give a reasonable representation of the transmission data, the BSRM approach was superior in its description of resonance effects (see Fig. 1 of [1]). The most important partial-wave contributions to the excitation cross sections of several states were also published, permitting a more sensitive experimental test of their proposed assignments. However, limitations on the BSRM model are expected where coupling to the target continuum is important.

Negative-ion resonance assignments have been proposed by both Sullivan et al. [3] and Zatsarriny and Bartschat [1]. They agree for features below $6.455 \mathrm{eV}$, with the exception of the $5.79 \mathrm{eV}$ structure assigned a $4 s 4 p^{2}{ }^{2} S$ resonance by experiment and a cusp by theory. Sullivan et al. assigned a ${ }^{2} D$ symmetry to the resonance near $4.3 \mathrm{eV}$ on the basis of energy-dependent elastic differential cross-section measurements, which are the only previous investigations of angledependent resonance behavior below the ionization threshold. Their other assignments were made using a graphical Rydberg binding energy method which identified regularities related to the principal quantum number $n$ in a comparative study of $\mathrm{Mg}, \mathrm{Zn}, \mathrm{Cd}$, and $\mathrm{Hg}$. Since then, Zatsarinny and Bartschat [1] have performed a detailed theoretical analysis and their assignments are included in Table I.

Our paper reports on the negative-ion resonances below the first ionization threshold observed in photon emission from electron-impact excited states of zinc. The energy resolution and statistics of our data establish precise energies and shapes in different excitation channels, and determine the width of some resonance structures.

\section{EXPERIMENT}

The excitation of free zinc atoms was studied using a versatile crossed-beam electron-impact spectrometer. Figure 1 is a schematic diagram showing the components of the apparatus essential for this study. A detailed description of the entire apparatus, which includes a scattered electron analyzer, will be presented elsewhere [11].
Electrons thermally emitted from a tungsten filament were energy selected using a $127^{\circ}$ cylindrical electrostatic monochromator and then focused into the interaction region. The electron optics were designed for low incident electron energies, between 3 and $20 \mathrm{eV}$. Since negative-ion structures typically have natural widths less than $100 \mathrm{meV}$, the spectrometer was tuned to optimize energy resolution and current, with less concern for constant transmission over energy ranges greater than $1 \mathrm{eV}$. No corrections were made for any energy-dependent transmission. The full width at half maximum of the incident electron beam Gaussian energy distribution $\Delta E_{1 / 2}$, was $80 \mathrm{meV}$. However, the resolution apparent in the data was not as good due to drifts in incident energy, which seem unavoidable when working with zinc and some other metal vapors [12]. The exact size of these energy drifts was unpredictable, but usually on the order of $20 \mathrm{meV}$ across a $12 \mathrm{~h}$ scan. Zinc accumulation within the apparatus restricted the total operating time of each data run to $\sim 100 \mathrm{~h}$, after which the electron optics and insulators required cleaning. It was therefore necessary to compromise between maximizing energy resolution and achieving a better overall data collection efficiency by maximizing the incident electron current. Measurements of weak spectral lines were done typically with $\Delta E_{1 / 2}=120 \mathrm{meV}$ and total data collection time varied from $4 \mathrm{~h}$ for strong to $36 \mathrm{~h}$ for weak lines.

Pellets of $99.999 \%$ purity zinc were vaporized within the main cavity of a molybdenum oven. A 150-mm-long diameter nozzle joined to the top of the oven directed free atoms into the interaction region, which was $7 \mathrm{~mm}$ from the $1 \mathrm{~mm}$ inner diameter, 12-mm-long nozzle tip. The oven and nozzle were independently heated by tightly enclosing copper cylinders with embedded twin filament Thermocoax heaters. Oven temperatures varied between $480{ }^{\circ} \mathrm{C}$ and $500{ }^{\circ} \mathrm{C}$ depending on the available signal, while the nozzle temperature was maintained $30{ }^{\circ} \mathrm{C}$ hotter to prevent blockage. A molybdenum shield covering the nozzle and a graphite soot coating on all nearby surfaces prevented the detection of thermal photons from the oven. A copper, liquid-nitrogen-cooled vapor trap was placed opposite the nozzle, reducing zinc deposition onto other components.

Decay photons were detected perpendicular to the incident electron beam, as shown in Fig. 1. Photons were wavelength selected using interference filters, discussed in detail for each transition in Sec. III, which allowed specific upper state selection for all transitions except $4 s 4 p{ }^{3} P_{1} \rightarrow 4 s^{2}{ }^{1} S_{0}$. However, only the near-threshold energy regions were free from cascade contributions. Photons were detected using an Electron Tubes 9863/350QB photomultiplier cooled to $-20^{\circ}$, which reduced the "dark noise" level to $\sim 4$ counts/s. The small dark noise and thermal photon contribution to the data was subtracted. Standard counting electronics and a PCbased acquisition system scanned the incident energy and processed the data.

Energy calibration was performed by detecting $636.2 \mathrm{~nm}$ photons (see Sec. III C below) as the incident electron energy was scanned across the steplike threshold of the $4 s 4 d{ }^{1} D_{2}$ state. The data were fitted against the BSRM integral cross section convoluted with a Gaussian experimental apparatus function, as shown in Fig. 9. This fit determined $\Delta E_{1 / 2}$ and the energy shift to within an estimated accuracy of 
TABLE I. Energies of structures observed in photon excitation functions for the $4 s 4 p{ }^{3} P_{1}, 4 s 4 p{ }^{1} P_{1}, 4 s 4 d{ }^{3} D_{1,2,3}, 4 s 4 d{ }^{1} D_{2}$, and $4 s 5 d^{3} D_{1,2,3}$ states below the first ionization threshold. Results from the BSRM theory [1] and previous transmission [3] and photon excitation function [6] experiments are presented for comparison. Energies given for the present experiment do not necessarily correspond to resonance energies, and a peak and an adjacent dip may correspond to a single resonance. The numbers in the leftmost column refer to the labels used to identify structures throughout this paper. $P$ indicates a peak, $D$ a dip, and $F$ a Fano fitted structure. Unless otherwise stated, the uncertainty in energy for the present experiment is $\pm 30 \mathrm{~m} \mathrm{eV}$. All energies are given in $\mathrm{eV}$.

\begin{tabular}{|c|c|c|c|c|c|c|c|c|}
\hline \multirow{2}{*}{$\begin{array}{l}\text { Structure } \\
\text { reference } \\
\text { number }\end{array}$} & \multirow[b]{2}{*}{$4 s 4 p^{3} P_{1}$} & \multicolumn{3}{|c|}{ Present experiment } & \multirow[b]{2}{*}{$4 s 5 d^{3} D_{1,2,3}$} & \multirow{2}{*}{$\begin{array}{l}\text { Photon } \\
\text { excitation } \\
\text { function }\end{array}$} & \multirow{2}{*}{$\begin{array}{c}\text { Transmission } \\
\text { experiment }\end{array}$} & \multirow[t]{2}{*}{ BSRM theory } \\
\hline & & $4 s 4 p{ }^{1} P_{1}$ & $4 s 4 d^{3} D_{1,2,3}$ & $4 s 4 d{ }^{1} D_{2}$ & & & & \\
\hline & & & & & & 4.36 & $4.25\left(4 s 4 p^{2}{ }^{2} D\right)$ & $4.234\left(4 s 4 p^{2}{ }^{2} D, \quad \Gamma=0.372\right)$ \\
\hline \multirow[t]{3}{*}{ (1) } & $F P 5.80^{\mathrm{a}}$ & $P 5.80$ & & & & & $5.79\left(4 s 4 p^{2}{ }^{2} S\right)$ & 5.796 (cusp) \\
\hline & & & & & & & $6.40\left(4 s 5 s^{2}{ }^{2} S\right)$ & \\
\hline & & & & & & & & $6.455\left(4 s 5 s^{2}{ }^{2} S, \quad \Gamma=0.075\right)$ \\
\hline (2) & & $D 7.01$ & & & & 7.18 & & \\
\hline \multirow[t]{4}{*}{ (3) } & & $P 7.44$ & & & & & $7.40(4 s 5 s 4 d)$ & \\
\hline & & & & & & & & $7.524\left(4 s 5 p^{2}{ }^{2} S, \quad \Gamma=0.051\right)$ \\
\hline & & & & & & 7.56 & $7.55\left(4 s 4 d^{2}\right)$ & $7.570\left(4 s 5 p^{2}{ }^{2} D, \quad \Gamma=0.051\right)$ \\
\hline & & & & & & & & $7.595\left(4 s 4 d 5 p^{2} P^{o}, \quad \Gamma=0.024\right)$ \\
\hline (4) & & $D 7.60$ & & & & & & $7.615\left(4 s 4 d 5 p^{2} F, \quad \Gamma=0.030\right)$ \\
\hline (5) & $F P 7.66^{\mathrm{a}}$ & $P 7.74^{\mathrm{b}}$ & & & & & $7.65\left(4 s 5 p^{2}{ }^{2} D\right)$ & $7.725\left(4 s 4 d 5 s^{2} D, \quad \Gamma=0.024\right)$ \\
\hline (6) & & & P7.87 & & & 7.80 & & $7.904\left(4 s 6 s^{2}{ }^{2} S, \Gamma=0.142\right)$ \\
\hline (7) & & & $D 8.11$ & $P 8.15$ & & 8.22 & & $8.122\left(4 s 5 d 6 s^{2} D, \Gamma=0.045\right)$ \\
\hline (8) & P8.28 & $D 8.25^{\mathrm{b}}$ & P8.24 & $D 8.29$ & & $8.33^{c}$ & & \\
\hline (9) & $D 8.39$ & $P 8.36$ & $D 8.40$ & $P 8.40$ & & & & $8.389\left(4 s 7 s^{2}{ }^{2} S, \quad \Gamma=0.184\right)$ \\
\hline & & & & & & & & $8.425\left(4 s 5 d 6 p^{2} P^{o}, \quad \Gamma=0.083\right)$ \\
\hline & & & & & & & & $8.438\left(4 s 5 d 6 p^{2} F^{o}, \quad \Gamma=0.08\right)$ \\
\hline$(10)$ & $P 8.54$ & & & $D 8.52$ & & & & $8.483\left(4 s 5 d 7 s^{2} D, \quad \Gamma=0.048\right)$ \\
\hline$(11)$ & & & & $P 8.64$ & $P 8.60$ & 8.60 & & \\
\hline (12) & $D 8.67$ & & $P 8.71^{\mathrm{b}}$ & & & & & \\
\hline (13) & $P 8.77$ & & & & $D 8.75^{\mathrm{b}}$ & & & \\
\hline (14) & & & $D 8.79$ & & & 8.80 & & \\
\hline
\end{tabular}

${ }^{\mathrm{a}}$ Fano profile fitted, energy uncertainty $\pm 24 \mathrm{~m} \mathrm{eV}$.

${ }^{\mathrm{b}}$ Weak structure, only observed after background subtraction. Energy uncertainty $\pm 60 \mathrm{~m} \mathrm{eV}$.

${ }^{\mathrm{c}}$ Mean value from different transitions.

$\pm 10 \mathrm{meV}$. Calibrations were performed before and after each scan, then averaged to account for energy drifts. The average difference between before and after calibrations was $20 \mathrm{meV}$, and so the energy due to energy drifts is estimated as $\pm 10 \mathrm{~m} \mathrm{eV}$. Without consideration of calibration and drift errors, the error in determining energies from the spectra obtained was $\pm 4 \mathrm{~m} \mathrm{eV}$ for Fano fitted structures, $\pm 10 \mathrm{~m} \mathrm{eV}$ or half the channel width for well-resolved structures, and $\pm 40 \mathrm{~m} \mathrm{eV}$ or twice the channel width for weaker structures indicated in Table I. Overall, the energy uncertainty was $\pm 24 \mathrm{meV}$ for Fano fitted structures, $\pm 30 \mathrm{meV}$ for other strong structures, and $\pm 60 \mathrm{meV}$ for weaker structures.

\section{RESULTS}

Photon excitation functions have been measured for the $4 s 4 p{ }^{3} P_{1}, 4 s 4 p{ }^{1} P_{1}, 4 s 4 d{ }^{3} D_{1,2,3}, 4 s 4 d{ }^{1} D_{2}$, and $4 s 5 d^{3} D_{1,2,3}$ states of zinc. Figure 2 shows an energy-level diagram and the wavelengths of transitions observed. For each state the incident electron energy was scanned from below the excitation threshold to approximately $10 \mathrm{eV}$. The lower and upper energy regions of the $4 s 4 p{ }^{3} P_{1}$ and $4 s 4 p{ }^{1} P_{1}$ states were scanned separately to optimize resolution and statistical accuracy.

Structures observed here are listed in Table I with $P$ indicating a peak and $D$ a dip, along with features in previous photon excitation functions [6,7], transmission experiments [3], and BSRM calculations [1]. Structures in the table are labeled with numbers which will be referred to throughout the text and in figures. The energies of isolated resonant structures were determined precisely by fitting a Fano profile to the data, using the method described in [5]. Resonance energies determined using this method are indicated by a letter $F$ and are also included in the corresponding figures. Above $7.4 \mathrm{eV}$ the overlap and possible interference of closely spaced negative-ion resonances resulted in complex shapes, making it difficult to determine true resonance energies. Instead, the resonance structures were isolated by sub- 


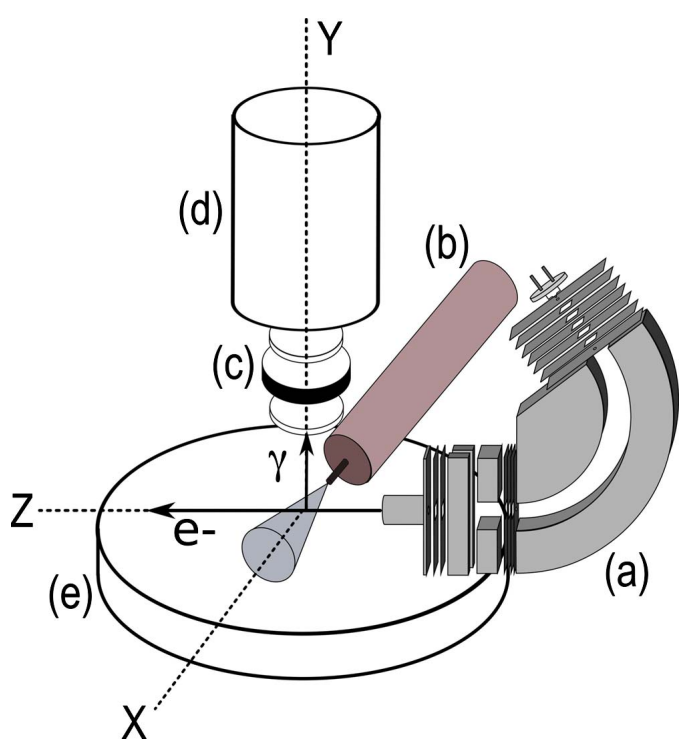

FIG. 1. (Color online) Schematic diagram of the experimental apparatus; (a) electron beam forming electrostatic monochrometer and optics; (b) zinc oven; (c) photon lenses and interference filter; (d) photomultiplier tube; and (e) liquid-nitrogen-cooled zinc vapor collection plate.

tracting a least-squares polynomial fit to the slowly varying non-resonance contribution, which is a similar technique to that used by Quéméner et al. [13]. Features in the resulting spectrum were then more easily identified and assigned energies. This approach did not determine the true number of resonances or their energies, so a comparison between the various data should not be made exclusively by inspection of Table I. Also, comparisons between different exit channels are complicated by varying resonance contributions and interference effects, which can result in a different appearance for the same resonance in different states.

\section{A. Excitation of the $4 s 4 p^{3} P_{1}$ state}

Figures 3 and 4 show observations of $\lambda=307.6 \mathrm{~nm}$ photons from the $4 s 4 p^{3} P_{1} \rightarrow 4 s^{2}{ }^{1} S_{0}$ transition. This spinforbidden excitation has a large cross section near its thresh-

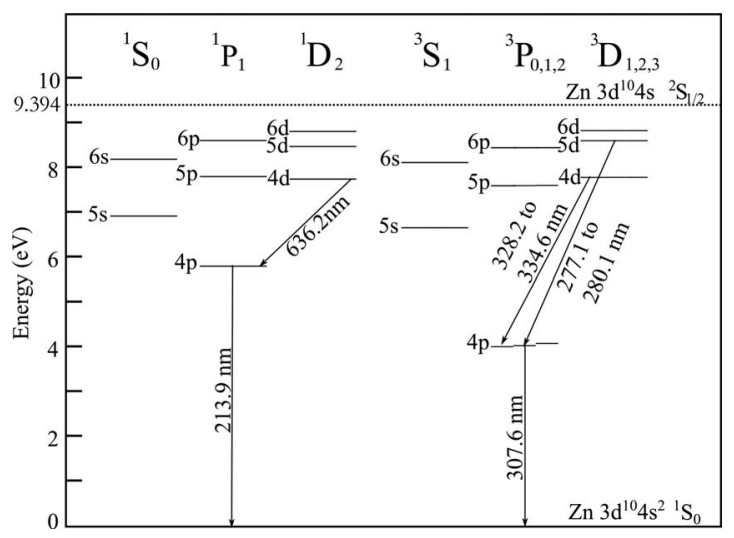

FIG. 2. Zinc energy level diagram, showing the transitions and wavelengths of interest for this work.

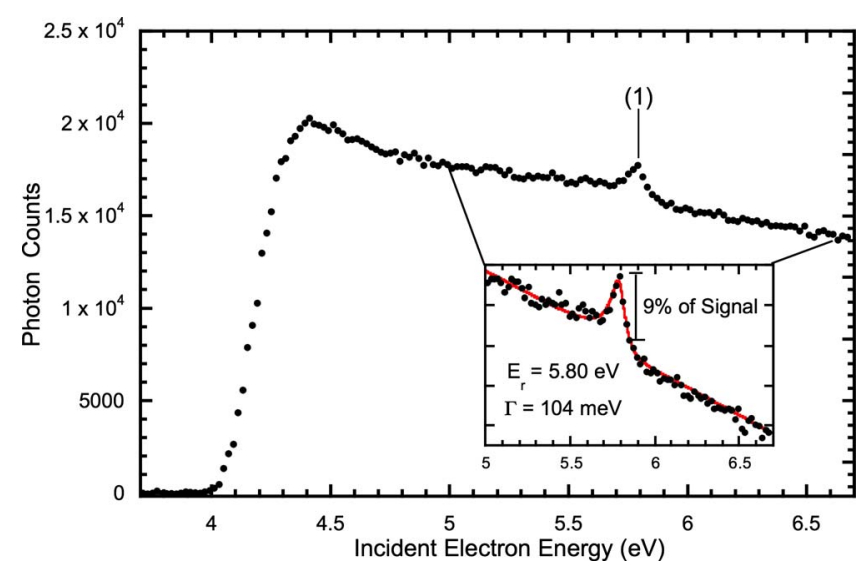

FIG. 3. (Color online) Photon excitation function for the $4 s 4 p{ }^{3} P_{1}$ state of zinc in the cascade-free region; $\boldsymbol{O}$, present data. Inset: - a combined polynomial background and Fano profile fit to the resonance structure which obtained the resonance energy and width displayed. Also shown is the resonance contribution as a percentage of the total signal.

old [1] and we observe rich resonance structure. Measurements above $6.655 \mathrm{eV}$ are strongly affected by cascades from the whole of the triplet manifold into this lowest triplet state. Effects in multiple negative-ion decay channels may be present simultaneously, which complicates analysis.

Figure 3 shows the cascade-free region up to the $4 s 5 s{ }^{3} S_{1}$-state threshold at $6.654 \mathrm{eV}$. A sharp feature, marked (1) in Fig. 3, is observed at $5.80 \mathrm{eV}$ in agreement with previous experimental [3] and theoretical work [1]. Observations from $6.5 \mathrm{eV}$ to the ionization threshold are shown in Fig. 4. Fano profiles were fitted to the isolated features in Figs. 3 and 4, which obtained the resonance energies and widths displayed. These widths result from the convolution of natural resonance widths with the finite experimental energy resolution. Resonance contributions as a percentage of the total signal are also shown.

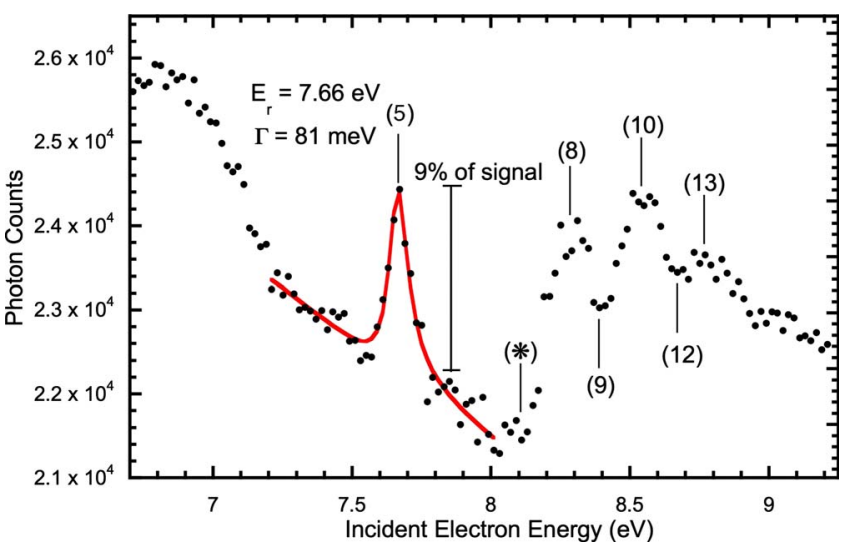

FIG. 4. (Color online) Photon excitation function for the $4 s 4{ }^{3} P_{1}$ state of zinc in the energy region from $6.7 \mathrm{eV}$ to the ionization threshold; $\boldsymbol{\bullet}$, present data; - , a combined polynomial background and Fano profile fit to the resonance structure which obtained the resonance energy and width displayed. Also shown is the resonance contribution as a percentage of the total signal. Cascade contributions are present, and are responsible for the enhancement above $8 \mathrm{eV}$. The threshold of the $4 s 6 s^{3} S$ state is marked (*). 


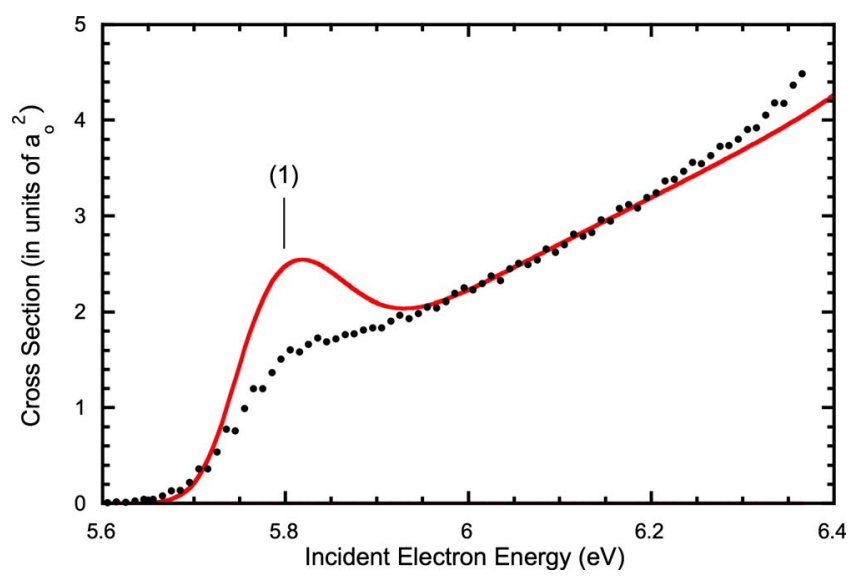

FIG. 5. (Color online) Integral cross section for the $4 s 4 p{ }^{1} P_{1}$ state of zinc in the energy region from threshold to $6.4 \mathrm{eV}$. - , present data normalized to theory; - BSRM theory, convoluted with $\Delta E_{1 / 2}=100 \mathrm{meV}$ apparatus function.

Cascades into $4 s 4 p^{3} P_{1}$ come from $4 s n d^{3} D_{1,2}$ and $4 s(n$ $+1) s^{3} S_{1}$ states, with $n=4$ likely to be the most significant. The $4 s 4 d{ }^{3} D_{1,2,3}$-state photon excitation function is presented in Sec. III C. A special situation arises relating to the excitation of the $4 s 6 s^{3} S_{1}$ state, the threshold for which is marked $(*)$ in Fig. 4. This state decays via the $4 s 6 s{ }^{3} S$ $\rightarrow 4 s 4 p{ }^{3} P_{1}$ transition with $\lambda=303.6 \mathrm{~nm}$ photons. The $\Delta \lambda$ $=12 \mathrm{~nm}$ bandwidth interference filter used had $18 \%$ transmission at $\lambda=307.6 \mathrm{~nm}$ and $13 \%$ transmission at $\lambda$ $=303.6 \mathrm{~nm}$, so the excitation of one atom into the $4 s 6 s^{3} S_{1}$ state had the potential to result in two photons being detected. The $8.11 \mathrm{eV}$ threshold for this state coincides with an enhancement in the photon yield. This enhancement could also have a strong cascade contribution from $n \geq 5$ and possibly $\ell \geq 2$ states.

The photon measurements shown in Fig. 4 cannot determine which structures are due to resonances decaying directly into the $4 s 4 p^{3} P_{1}$ state, and which appear through cascades. In the cascade effected region, all structures in the $4 s 4{ }^{3} P_{1}$ excitation function, except the dip at $8.67 \mathrm{eV}$ marked (12) in Fig. 4, have a corresponding feature in the higher triplet state excitation functions reported here. Electron excitation function measurements are not effected by cascades, and are currently underway to resolve this ambiguity. Preliminary results for $4 s 4 p^{3} P_{0,1,2}$ differential electron excitation functions [11] show some structure in this region.

\section{B. Excitation of the $4 s 4 p{ }^{1} P_{1}$ state}

Figures 5 and 6 show observations of $\lambda=213.8 \mathrm{~nm}$ photons from the $4 s 4 p{ }^{1} P_{1} \rightarrow 4 s^{2}{ }^{1} S_{0}$ transition. Photons were selected using an interference filter with $16 \%$ transmission at this wavelength. Cascade contributions from the $4 s n s{ }^{1} S_{0}$ and 4 snd ${ }^{1} D_{2}$ states are present from $6.917 \mathrm{eV}$ onwards. Generally speaking, resonance contributions as a fraction of total signal are small compared to the other states studied. This may be due to the large cross section for direct $4 s 4 p{ }^{1} P_{1}$ excitation.

Figure 5 compares the normalized present data with the BSRM $4 s 4 p{ }^{1} P_{1}$ integral cross section from threshold to 6.4
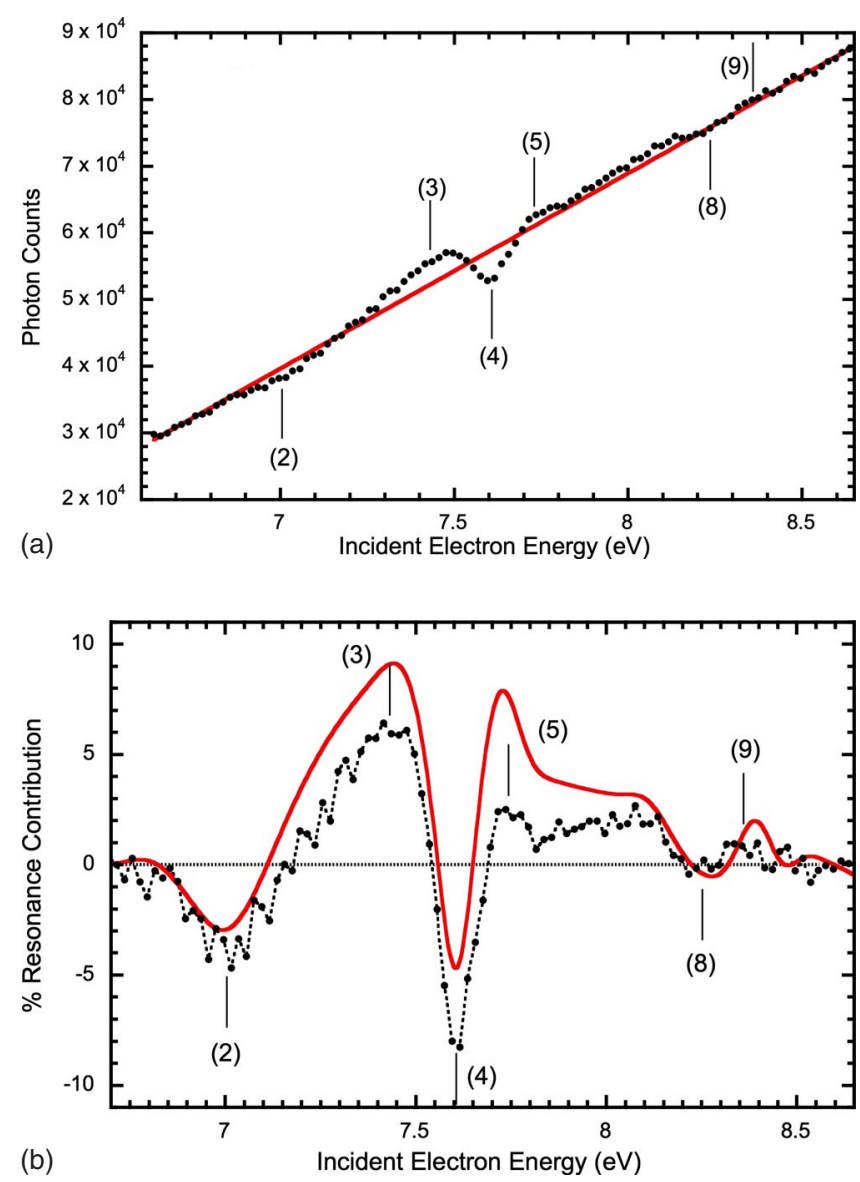

FIG. 6. (Color online) Photon excitation function for the $4 s 4 p{ }^{1} P_{1}$ state of zinc in the energy region from 6.5 to $8.5 \mathrm{eV}$. From $6.917 \mathrm{eV}$ onwards cascade contributions are present. (a) $\bullet$, present data; - , fit to the background obtained using the method described in the text. (b) - The resonance contribution to the present data, obtained by subtracting the estimated background; - the resonance contribution to the estimated BSRM emission cross section, obtained by subtracting a background estimated in the same manner as for the experimental data (see the text for details).

$\mathrm{eV}$. The calculations have been convoluted with a $\Delta E_{1 / 2}$ $=100 \mathrm{meV}$ apparatus function. This was determined to be the best estimate of resolution for this particular scan by comparing experiment and the theory convoluted with a range of apparatus functions. BSRM calculations contain a structure at $5.79 \mathrm{eV}[1]$, which has been assigned as a cusp on the basis of a time delay analysis. The proximity of the feature marked (1) in Fig. 3 to the $4 s 4 p{ }^{1} P_{1}$-state threshold supports this conclusion. A cusplike structure has also been observed in the elastic energy-dependent differential cross section at $5.79 \mathrm{eV}$ [11]. Figure 5 shows a shoulder structure, marked (1), which suggests a threshold feature. However, the feature is much less prominent in the experimental data than in the convoluted theory.

In the higher energy region shown in Fig. 6, there is a dominant dip at $7.60 \mathrm{eV}$, marked (4), within a complex structure. BSRM theory predicts resonances at 7.570, 7.595, and $7.615 \mathrm{eV}$, with widths and assignments shown in Table I. BSRM integral cross sections for the $4 s 4 p{ }^{1} P_{1}, 4 s 4 d{ }^{1} D_{2}$ and $4 s 5 s{ }^{1} S_{0}$ states were summed, convoluted with a $\Delta E_{1 / 2}$ 
$=80 \mathrm{meV}$ apparatus function then normalized to the present measurements. Adding theoretical cascade contributions made significant modifications to both the general shape and prominence of resonance features. The maximum $4 s 5 s{ }^{1} S_{0}$ contribution to the total emission cross section was $12 \%$ at $7.5 \mathrm{eV}$, while for $4 s 4 d{ }^{1} D_{2}$ it was $8 \%$ at $7.9 \mathrm{eV}$, assuming these states decay only to the $4 s 4 p{ }^{1} P_{1}$ state. The theoretical resonance contribution was isolated by subtracting a fit to the smoothly varying background, as was done for the experimental data. This allowed the direct comparison of crosssection shapes presented in Fig. 6(b). The general shapes of the experimental and theoretical structures are in excellent agreement, with the average energy discrepancy between corresponding features being $17 \mathrm{meV}$.

\section{Excitation of the $4 s 4 d^{3} D_{1,2,3}$ state}

Transitions from the various fine structure components of $4 s 4 d^{3} D_{1,2,3} \rightarrow 4 s 4 p{ }^{3} P_{0,1,2}$ give rise to six closely spaced lines with wavelengths between 328.2 and $334.6 \mathrm{~nm}$. The interference filter used was centered on $334.2 \mathrm{~nm}$ with $14 \%$ transmission at $328.2 \mathrm{~nm}$ and $34 \%$ transmission at $334.6 \mathrm{~nm}$, so all of these lines were detected but with different efficiencies. The photon excitation function for this state is shown in Fig. 7. A steep onset to the experimental data peaks $90 \mathrm{meV}$ above the $7.78 \mathrm{eV}$ threshold, and is followed by a second sharp peak at $8.24 \mathrm{eV}$, marked (8) in Fig. 7. Both peaks are observed in the cascade-free energy region, which extends to the $4 s 6 p^{3} P_{0,1,2}$-state threshold at $8.44 \mathrm{eV}$. $L \geq 3$ states within the triplet manifold will cascade preferentially through $4 s 4 d^{3} D_{1,2,3}$, and there will also be contributions coming through 4 snp ${ }^{3} P_{0,1,2}$ states. Resonance contributions to this and all other $D$ states studied are strong, and generally comprise a greater proportion of the total signal than for the $P$ states studied.

Both the $4 s 4 d^{3} D_{1,2,3}$ and $4 s 4 p{ }^{3} P_{1}$ photon excitation functions exhibit a peak at $8.26 \mathrm{eV}$ and a dip at $8.40 \mathrm{eV}$, marked (8) and (9) in Figs. 4 and 7. Conversely, the $4 s 4 p{ }^{1} P_{1}$ and $4 s 4 d{ }^{1} D_{2}$ states have a dip at $8.26 \mathrm{eV}$ and a peak at 8.38 eV. A comparison of Figs. 7 and 4 also indicates that the near-threshold excitation of $4 s 4 d^{3} D_{1,2,3}$ has little effect on the $4 s 4 p{ }^{3} P_{1}$ photon excitation function. The sharp onset of the $4 s 4 d^{3} D_{1,2,3}$ state ends $200 \mathrm{meV}$ before the sharp onset of cascades into $4 s 4 p{ }^{3} P_{1}$ begins. Perhaps the vertical onset and threshold peak in $4 s 4 d^{3} D_{1,2,3}$ is due entirely to the $J=3$ state, which does not decay to $4 s 4 p^{3} P_{1}$.

\section{Excitation of the $4 s 5 d^{3} D_{1,2,3}$ state}

The six lines resulting from the $4 s 5 d^{3} D_{1,2,3}$ $\rightarrow 4 s 4 p^{3} P_{0,1,2}$ transition are spread from 275.6 to $280 \mathrm{~nm}$. All of these wavelengths were detected by using an interference filter centered on $283 \mathrm{~nm}$ with $5 \%$ transmission at 275.6 $\mathrm{nm}$ and $16 \%$ transmission at $280 \mathrm{~nm}$. Figure 8 shows the $4 s 5 d^{3} D_{1,2,3}$ photon excitation function measured from threshold to $10 \mathrm{eV}$. The threshold behavior and general shape of the excitation function is very similar to the $4 s 5 d^{3} D_{1,2,3}$ state. A sharp onset at threshold is followed by a dip at 8.75 eV marked (13) in Fig. 8. Cascade contributions will be
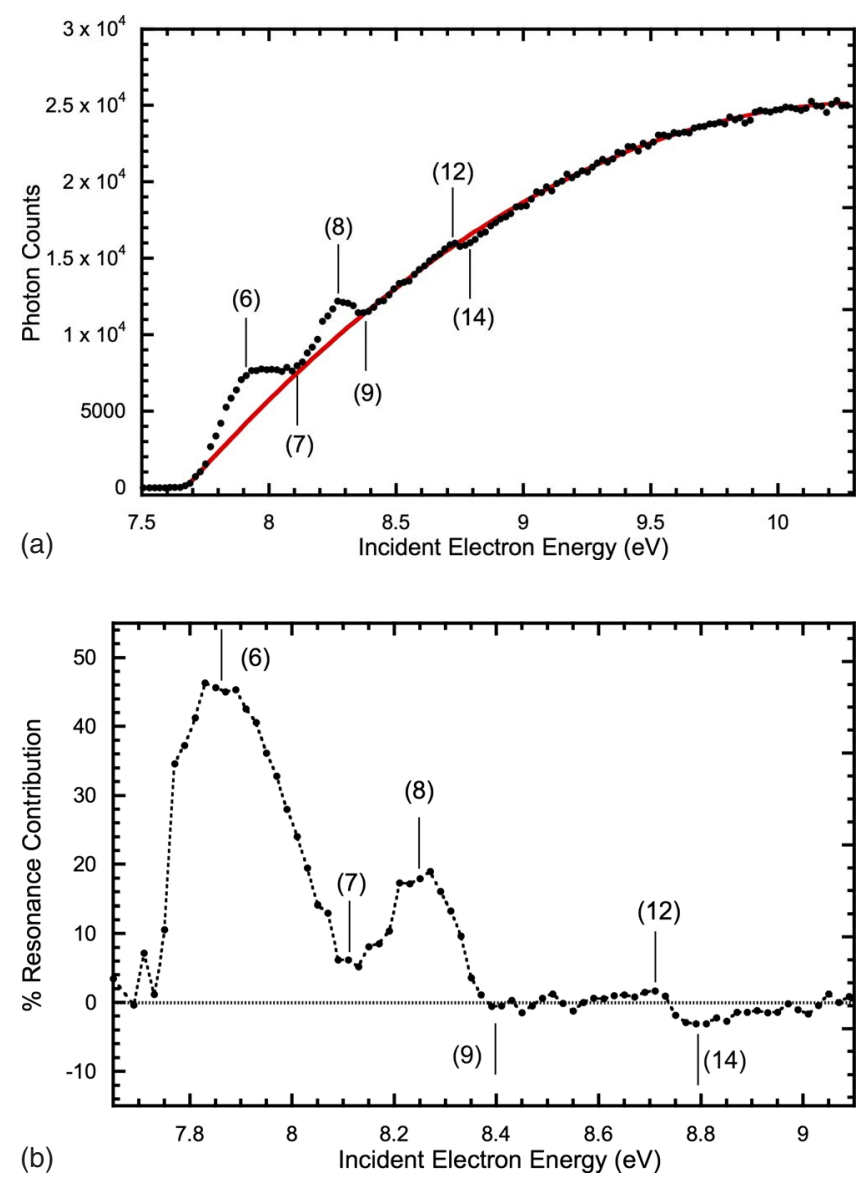

FIG. 7. (Color online) Photon excitation function for the $4 s 4 d^{3} D_{1,2,3}$ state of zinc in the energy region from threshold to 10 eV. From $8.44 \mathrm{eV}$ onwards cascade contributions are present. Triplet states with $L \geq 3$ cascade largely into this state, and there will also be contributions from $4 \operatorname{snp}{ }^{3} P_{1,2,3}$ states for $n \geq 6$. (a) present data; - a fit to the background obtained using the method described in the text. (b) - - , the resonance contribution to the present data, obtained by subtracting the estimated background.

present almost from threshold, but are expected to be less significant than for the lower-lying $4 s 4 d^{3} D_{1,2,3}$ state.

\section{E. Excitation of the $4 s 4 d{ }^{1} D_{2}$ state}

Figure 9 shows observations of $\lambda=636.2 \mathrm{~nm}$ photons from the $4 s 4 d{ }^{1} D_{2} \rightarrow 4 s 4 p^{1} P_{1}$ transition from threshold to 10 $\mathrm{eV}$. The photons were selected by an interference filter with $65 \%$ transmission at this wavelength. Since 4 snp ${ }^{1} P_{1}$ states will decay predominantly to the ground state, cascade contributions come mostly from $4 \operatorname{snf}{ }^{1} F_{3}$ states, with the $n=4$ threshold at $8.53 \mathrm{eV}$. Both theory and experiment show a steplike onset at threshold, and so a convoluted step function was included in the background fit subtracted from the raw data.

Strong resonances are clearly visible up to $8.64 \mathrm{eV}$, producing alternating peaks and dips. The energies of the lowest three structures correspond well with structures in $4 s 4 d^{3} D_{1,2,3}$, however, the peaks in the $4 s 4 d{ }^{1} D_{2}$ excitation function appear as dips in the $4 s 4 d^{3} D_{1,2,3}$ function, and vice versa. This observation indicates different interference be- 

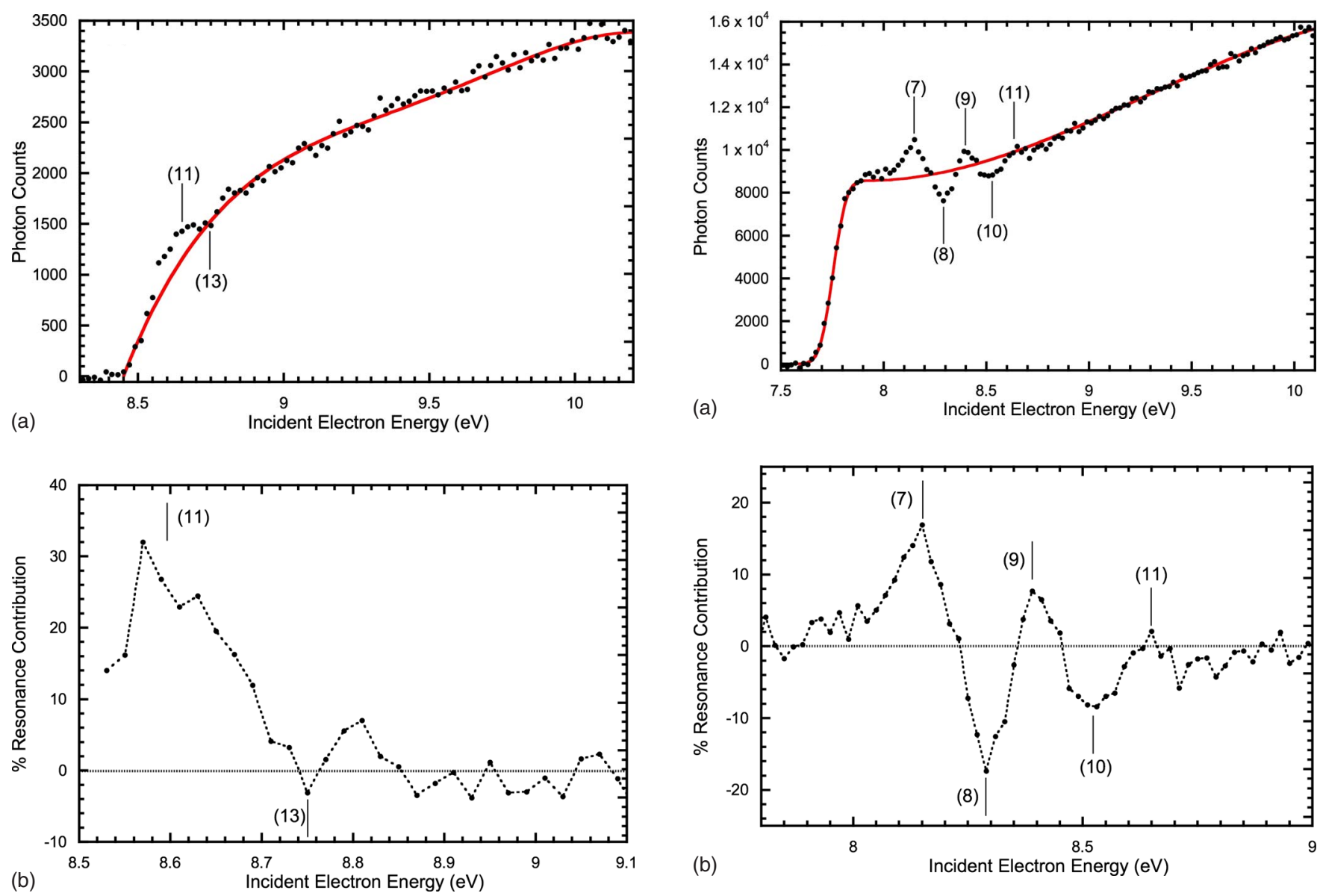

FIG. 8. (Color online) Photon excitation function for the $4 s 5 d^{3} D_{1,2,3}$ state of zinc in the energy region from threshold to 10 $\mathrm{eV}$. Cascade contributions will be present almost from threshold, but are expected to be less significant than for the lower-lying $4 s 4 d^{3} D_{1,2,3}$ state. (a) $\bullet$, present data; - a fit to the background obtained using the method described in the text. (b) - - -, the resonance contribution to the present data, obtained by subtracting the estimated background.

tween the resonance and the continuum in different channels, and possibly the presence of more resonances than indicated by just the number of peaks.

Figure 9(c) compares the present data with the BSRM calculations, convoluted with a $\Delta E_{1 / 2}=80 \mathrm{meV}$ apparatus function. A peak in the BSRM integral cross section due to the $8.122 \mathrm{eV}$ resonance corresponds almost exactly with an experimental feature at $8.15 \mathrm{eV}$, marked (7) in Fig. 9. There is good agreement between theory and experiment up to 8.30 $\mathrm{eV}$, after which they start to diverge.

\section{DISCUSSION}

Previous photon excitation function measurements [6,7] were not analyzed in as much detail as the data presented here. Electron transmission studies [3] present the derivative of the transmitted current with respect to energy and include effects from all scattering channels, so resonance shapes and energies are not always easy to compare with photon emission data. The transmission experiment did not extract reso-

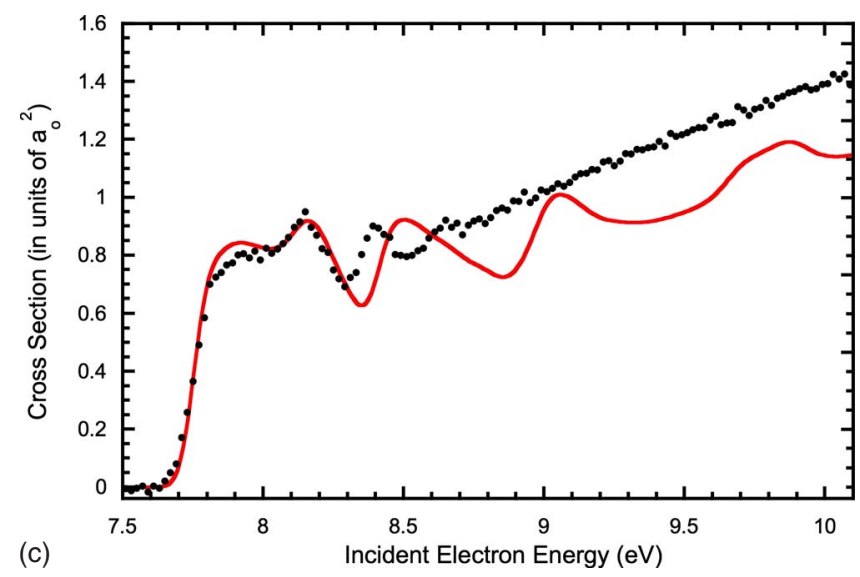

FIG. 9. (Color online) Photon excitation function for the $4 s 4 d{ }^{1} D_{1}$ state of zinc in the energy region from threshold to $10 \mathrm{eV}$. Since $4 \operatorname{sn} p{ }^{1} P_{1}$ states will decay predominantly to ground, cascade contributions come from $4 s n f^{1} F_{3}$ states, starting at $8.53 \mathrm{eV}$. (a) $\bullet$, present data; - a fit to the background obtained using the method described in the text. (b) --, The resonance contribution to the present data, obtained by subtracting the estimated background. (c) Integral cross section from threshold to $10 \mathrm{eV}$. $\mathbf{0}$, present data normalized to theory; - BSRM theory convoluted with a $\Delta E_{1 / 2}$ $=80 \mathrm{meV}$ apparatus function.

nance energies above $7.65 \mathrm{eV}$, even though structures were observed at higher energies. Bearing this in mind, the agreement between the present and these previous experiments is 
within expected limits of deviation. The present data do not agree with $4 s 4 p{ }^{3} P_{0,1,2}$ and $4 s 4 p{ }^{1} P_{1}$ integral cross-section measurements [8], the only existing scattered electron study which is relevant here. This previous study detected the excitation of all $4 s 4 p^{3} P_{0,12}$ state $J$ levels, but the resulting curve does not show any structures below $6.5 \mathrm{eV}$ corresponding to feature (1) shown in Fig. 3. The $4 s 4 p{ }^{1} P_{1}$ cross section shows resonant structures below the ionization threshold in agreement with [6]. However, the relative resonance contributions of up to $50 \%$ are much greater than in the present experiment.

The present data agree well with BSRM [1] resonance energies and profiles below $\sim 8 \mathrm{eV}$, and near-threshold cross-section behavior for the $4 s 4 p{ }^{1} P_{1}$ and $4 s 4 d{ }^{1} D_{2}$ states, shown in Figs. 5 and 9(c). For $4 s 4 p{ }^{1} P_{1}$ excitation, there is good agreement between the experimental and theoretical resonance contributions extending to $8.5 \mathrm{eV}$, as can be seen in Fig. 6. BSRM calculations also reproduced the transmission curve in [3] very well. However, Fig. 9(c) shows the BSRM cross section for $4 s 4 d{ }^{1} D_{2}$-state excitation fluctuating relative to the experimental shape above $8.2 \mathrm{eV}$, indicating a loss of accuracy expected for higher energies. Zatsarinny and Bartschat note that their approach is most reliable in the near-threshold regime, and has limitations for optically forbidden transitions.

For helium, $1 s 3 s^{3} S_{1}$ and $1 s 3 s^{1} S_{0}$-state integral crosssection studies [14] showed a remarkable agreement between experiment and 69 state $B$-spline $R$-matrix (BSR-69) calculations (69 coupled states with $S, P, D$, and $F$ symmetry). This theoretical method produced both the general crosssection shape and the finer details seen by the experiment throughout the $n=3,4$, and 5 resonance manifold, demonstrating its excellent predictive capability. A breakdown of the frozen-core model and stronger electron correlation effects are probably responsible for the deviations from the present zinc experiment.

A comparison of the present data with BSRM partialwave calculations permits a discussion of partial-wave compositions and resonance assignments. This will be restricted here to $4 s 4 p{ }^{3} P_{1}$ and $4 s 4 p{ }^{1} P_{1}$ excitation below $8.4 \mathrm{eV}$, where confidence in the theory is greatest. The resonance energies and widths predicted by theory suggest a great deal of overlap and possibly interference between adjacent resonances. These interference effects are not considered here. For $4 s 4 p{ }^{3} P_{1}$ excitation, the resonance (5) at $7.66 \mathrm{eV}$ shown in Fig. 4 corresponds best in shape and energy to a resonance in the ${ }^{2} D^{e}$ partial wave (see [1] Fig. 3). This confirms the predicted dominance of the ${ }^{2} D^{e}$ partial wave and suggests the $4 s 4 d 5 s^{2} D$ resonance contributes most strongly to this feature.

The $4 s 4 p{ }^{1} P_{1}$ data shown in Fig. 6(b) has its strongest feature at $7.60 \mathrm{eV}$, marked (4). Figure 4 of [1] shows this resonance in the ${ }^{2} F^{o}$ partial wave, suggesting a $4 s 4 d 5 p{ }^{2} F$ assignment. Likewise, the peak in the experimental data at
$7.44 \mathrm{eV}$, marked (3) in Fig. 6, could be due to the $4 s 4 p^{2}{ }^{2} \mathrm{D}$ resonance at $7.57 \mathrm{eV}$, and the peak at $8.36 \mathrm{eV}$, marked (9) in Fig. 6, could be due to a combination of the $4 s 7 s^{2}{ }^{2} S$ and $4 s 5 d 6 p{ }^{2} F$ resonances.

There are numerous instances in the present data where features in a triplet-state excitation function also exist in a singlet-state excitation function, but have the opposite appearance. The strongest examples are the adjacent structures at $\sim 8.26 \mathrm{eV}$ and $\sim 8.39 \mathrm{eV}$, where the $4 s 4 p^{3} P_{1}$ and 4 snd ${ }^{3} D_{1,2,3}$ states have a peak followed by a dip, while the $4 s 4 p{ }^{1} P_{1}$ and $4 s 4 d{ }^{1} D_{2}$ states have a dip then a peak. Table I shows that only one feature seen in both a singlet and a triplet state does not have this property.

\section{CONCLUSION}

We have presented a detailed study of temporary negative-ion formation in zinc and its effect on photon emission. High resolution photon excitation functions were measured for a selection of singlet and triplet states. As negative ions are highly correlated systems, their study in zinc offers an opportunity to test when the frozen core approximation is not applicable. A wealth of resonance structure is apparent in the present data, which shows the importance of electron correlations in this atom. Resonance contributions as a proportion of the total cross section were higher for $D$ states than for $P$ states, implying that the transfer of two units of orbital angular momentum is made more probable by resonance formation. The experimental resonance shapes confirmed some theoretical resonance assignments and dominant partial waves predictions for the $4 s 4 p^{3} P_{1}$ and $4 s 4 p{ }^{1} P_{1}$ states below $8.40 \mathrm{eV}$. Fine details in the powerful BSRM model start to deviate from experiment above $8.20 \mathrm{eV}$ for the nonoptically allowed $4 s 4 d{ }^{1} D_{2}$ state. This indicates the importance of coupling to the continuum in this instance.

Considering the difficulty of achieving better energy resolution and stability over long data collection times, we estimate that there is little chance of improvement for the present experiment. However, as the quality of theoretical models increase, comparisons of convoluted theoretical and experimental data will be excellent tests of predictive capacity. Larger $R$-matrix calculations by the same authors are planned, and some encouraging preliminary results already exist. These aim to more accurately calculate fine details in the cross sections up to and possibly above the ionization threshold.

\section{ACKNOWLEDGMENTS}

This work was supported by the Australian Research Council and the University of Western Australia. We are grateful to $\mathrm{O}$ Zatsarinny and $\mathrm{K}$ Bartschat for their data in electronic form. We also thank Steve Key for his excellent technical support. 
[1] O. Zatsarinny and K. Bartschat, Phys. Rev. A 71, 022716 (2005).

[2] S. J. Buckman and C. W. Clark, Rev. Mod. Phys. 66, 539 (1994).

[3] J. P. Sullivan, P. D. Burrow, D. S. Newman, K. Bartschat, J. A. Michejeda, R. Panajotovic, M. Moghbelalhossein, R. P. McEachran, and S. J. Buckman, New J. Phys. 5, 159.1 (2003).

[4] D. S. Newman, M. Zubek, and G. C. King, J. Phys B 18, 985 (1985).

[5] L. Pravica, D. Cvejanovic, J. F. Williams, and S. A. Napier, Phys. Rev. A 75, 030701(R) (2007).

[6] O. B. Shpenik, I. P. Zapesochnyi, V. V. Sovter, E. E. Kontrosh, and A. N. Zavilopulo, Sov. Phys. JETP 38, 898 (1974).

[7] O. B. Shpenik, M. M. Erdevdy, and J. E. Kontros, Radiat.
Phys. Chem. 76, 587 (2007).

[8] O. B. Shpenik, I. V. Chernishova, and J. E. Kontros, Radiat. Phys. Chem. 68, 277 (2003).

[9] P. D. Burrow, J. A. Micheida, and J. Comer, J. Phys B 9, 3225 (1976).

[10] O. Zatsarinny and C. F. Fischer, J. Phys. B 33, 313 (2000).

[11] S. A. Napier, D. Cvejanovic, J. F. Williams, and L. Pravica (unpublished).

[12] A. J. Murray, and D. Cvejanović, J. Phys. B 36, 4875 (2003).

[13] J. J. Quéméner, C. Paquet, and P. Marmet, Phys. Rev. A 4, 494 (1971).

[14] S. Stepanovic, M. Minic, D. Cvejanović, J. Jureta, J. Kurepa, S. Cvejanovic, O. Zatsarinny, and K. Bartschat, J. Phys. B 39, 1547 (2006). 